\title{
ANÁLISE DO USO DE BEBIDAS ALCOÓLICAS POR ESTUDANTES DE MEDICINA DE UMA INSTITUIÇÃO PRIVADA DE ENSINO
}

\author{
Eduardo Brito Souza Nóbrega ${ }^{\text {* }}$ \\ Lucas Lopes Fernandes "I \\ Arthur Gaia Duarte Peixoto III \\ Tânia Regina Ferreira Cavalcanti ${ }^{\text {IV }}$
}

\begin{abstract}
RESUMO
O excesso no uso do álcool pode causar danos nas mais diversas esferas da vida do ser humano, constituindo-se em um fator de risco para vários problemas de saúde e comportamental. Existem alguns grupos populacionais mais susceptíveis ao uso abusivo do álcool, tais como profissões de grande tensão emocional e pessoas sob grande estresse. O objetivo desse artigo é analisar o padrão do uso de álcool entre estudantes de medicina. Trata-se de uma pesquisa de campo de caráter descritivo com abordagem quantitativa. A população são todos 815 discentes entre o primeiro e o oitavo período da Faculdade de Medicina Nova Esperança. A amostra foi calculada com erro amostral de $11 \%$ e o nível de confiança de $95 \%$, chegando ao resultado de 73 estudantes. Esse valor foi arredondado para 80 com o objetivo de utilizar 10 discentes de cada período e obter uma amostra mais representativa do grupo populacional. Para a coleta de dados foi aplicado um questionário semiestruturado. De acordo com os resultados, $93,75 \%$ dos participantes relataram já ter provado bebidas alcoólicas e $44 \%$ destes mantêm o hábito de beber. $78 \%$ das mulheres e $44,8 \%$ dos homens tiveram o primeiro contato entre 15-18 anos. A média de alunos sem hábito de beber é de 6,25 nos primeiros 4 períodos e 4,25 nos 4 últimos. Estes dados sugerem um aumento no índice de alunos com hábito de beber ao longo dos períodos. Conclui-se que geralmente o primeiro contato com o álcool é na adolescência e, apesar da grande maioria já ter experimentado bebidas alcóolicas, a maior parte dos alunos não mantiveram o hábito de beber. Foi possível observar que o hábito de beber é mais prevalente em alunos de períodos mais avançados do que os estudantes que ingressaram mais recentemente no curso.
\end{abstract}

PALAVRAS-CHAVE: Alcoolismo. Estudantes de Medicina. Epidemiologia.

Acadêmico de Odontologia. Faculdade Nova Esperança (FAMENE).

ORCID: 0000-0002-7353-085X

Autor correspondente: eduardo.bsn@hotmail.com

Acadêmico de Odontologia. Faculdade Nova Esperança (FAMENE). "I

ORCID: 0000-0002-1644-8104

Acadêmico de Odontologia. Faculdade Nova Esperança (FAMENE). ${ }^{\text {II }}$

ORCID: 0000-0003-0944-2481

Docente da Faculdade Nova Esperança (Facene). IV

ORCID: 0000-0003-3084-6720 


\section{INTRODUÇÃO}

O álcool é a substância psicoativa mais utilizada em grande parte do mundo. $O$ início de uso ocorre entre os 10 e os 19 anos de idade, período caracterizado por alterações fisiológicas e sociais que podem aumentar a susceptibilidade ao uso abusivo. $\mathrm{O}$ alcoolismo pode ser responsável por vários danos às esferas sociais, emocional e orgânica. $\mathrm{O}$ uso cotidiano de álcool, desde a juventude, pode influenciar hábitos da vida adulta, sendo um fator de risco para problemas de saúde posteriores, além de provocar um risco maior de o indivíduo abusar dessas bebidas ao longo de sua vida., ${ }^{1,2}$

Um indivíduo sob efeito do álcool tem maior pré-disposição a adotar comportamentos de risco, podendo causar acidentes de trânsito e domésticos, gerando maior morbimortalidade a si e a comunidade ao seu redor. Pode também desenvolver maior absenteísmo acadêmico e laboral, o que irá prejudicar a sua formação e diminuir a sua produtividade. 3,4

\section{MATERIAL E MÉTODOS}

Trata-se de uma pesquisa de campo de caráter descritivo com abordagem quantitativa sobre o uso de álcool pelos estudantes de Medicina. O projeto de pesquisa foi submetido a análise pelo Comitê de Ética em Pesquisa FACENE/FAMENE, sendo aprovado, com número do CAEE: 59466016.6.0000.5179.

A pesquisa foi realizada na Faculdade de Medicina Nova Esperança (FAMENE), no município de João Pessoa, no estado da Paraíba. A população constitui-se de todos os discentes entre o primeiro e o oitavo período da referida Instituição, totalizando 815 alunos. A amostra foi calculada com erro amostral de $11 \%$ e o nível de confiança de $95 \%$, chegando ao
O ambiente acadêmico permite o crescimento pessoal e intelectual, sendo importante para estabelecer bases para uma vida profissional bem estruturada. Entretanto, esse ambiente é um grande estressor e pode facilitar ao abuso de várias drogas. Outro facilitador é o marketing de festas e comemorações que instigam o uso não saudável da bebida. 4

O álcool reduz a expectativa de vida de várias maneiras, não somente por provocar doenças como câncer, aterosclerose e hepatopatias, mas também predispõe situações de risco à vida, tais como a condução de veículos sobre efeito de entorpecentes, relações sexuais sem proteção, violência e outras. 4

O objetivo desse artigo é analisar o padrão do uso de álcool por estudantes de medicina, estudando o perfil do aluno que faz uso abusivo dessa substância e a relação entre o etilismo e o estresse da vida acadêmica.

resultado de 73 alunos. Este valor foi arredondado por convenção 80 para utilizar 10 discentes de cada período para obter uma amostra mais representativa do grupo populacional. Para seleção da referida amostra foram considerados os seguintes critérios de inclusão: discentes com mais de 18 anos, devidamente matriculados, que concordassem em participar da pesquisa, mediante assinatura no Termo de Consentimento Livre e Esclarecido - TCLE.

Um questionário foi aplicado para coletar os dados composto de duas partes: Parte I - dados de identificação dos participantes e Parte II - dados relacionados à temática Etilismo entre Estudantes de Medicina. A coleta 
ocorreu nos turnos da manhã e tarde do mês de março e abril de 2017, sendo dividida em dois momentos: os alunos receberam o TCLE e foram informados sobre a sigilosidade; uma breve explicação pelos pesquisadores sobre o questionário e a entrega do mesmo. A pesqui- sa foi analisada com foco no método quantitativo. Os dados foram analisados e tabulados estatisticamente, com o auxílio de um pacote estatístico SPSS (Versão 18), tabelas foram criadas para melhor análise e apresentação dos dados.

\section{RESULTADOS}

Participaram da pesquisa 80 estudantes de medicina, dos sexos masculino e feminino, sendo 10 alunos de cada um dos oito primeiros períodos, na faixa etária entre 18 e 32 anos, com predomínio de $81,25 \%$ dos estudantes entre 18 e 24 anos. 95\% dos participantes são solteiros, $37,5 \%$ dos entrevistados possuem renda familiar acima de 10 salários mínimos, 22,5\% de 7-9 SM, 23,75\% entre 4-6 SM. Quarenta e cinco pessoas afirmaram residir com os pais, 16 moram sozinhos, 5 com amigos, 4 com outros familiares, 4 com seus cônjuges.

Com relação a já ter experimentado bebidas alcoólicas, 4 pessoas afirmaram nunca terem ingeridos e 76, (95\%) dos entrevistados, relataram ter provado bebidas alcoólicas, sendo que 29 mantiveram o hábito de beber.

A tabela 1 demonstra a idade, o local e/ ou de quem as mulheres estavam acompanhadas quando tiveram o primeiro contato com álcool. De acordo com a análise dos valores, a idade mais frequente está entre os 15 a 18 anos (51,06\%), o local onde ingeriram bebida a primeira vez, na maioria das vezes, é a casa de amigos e, em segundo lugar, continua com a presença de amigos, mas em boates/bares (14,89\%).

TABELA 1- Primeiro contato com álcool das estudantes do sexo feminino.

\begin{tabular}{c|c|c|cc|c}
\hline \hline \multicolumn{6}{c}{ Uso de álcool } \\
\hline \hline Idade & Casa de Amigos & Com amigos em boate/bar & Com os Pais & Com familiares & TOTAL \\
\hline \hline$<12$ anos & - & - & 1 & 1 & 2 \\
$12-14$ anos & 1 & 1 & - & 1 & 3 \\
$15-18$ anos & 24 & 7 & 2 & 4 & 37 \\
$19-21$ anos & 1 & 2 & - & 1 & 4 \\
$>21$ anos & 1 & - & - & - & 1 \\
Total & 27 & 10 & 3 & 7 & 47 \\
\hline \hline
\end{tabular}

Já a tabela 2 visualizamos a idade, o local e/ou de quem os homens estavam acompanhados no primeiro contato com bebidas alcoólicas. De acordo com a tabela, os valores encontrados são mais homogêneos, a companhia mais frequente foi com amigos em boate/bar com 11 respostas (37,93\%), em segundo lugar está a opção "casa de amigos" com 8 respostas (27,58\%), em terceiro foi com os familiares com 6 participantes $(20,68 \%)$ e 
em quarto "com os pais" (13,79\%).

TABELA 2- Primeiro contato dos participantes do sexo masculino com álcool

\begin{tabular}{c|c|c|cc|c}
\hline \hline \multicolumn{6}{c}{ Uso de álcool } \\
\hline \hline Idade & Casa de Amigos & Com amigos em boate/bar & Com os Pais & Com familiares & TOTAL \\
\hline \hline$<12$ anos & - & - & 2 & 1 & 3 \\
$12-14$ anos & 3 & 4 & 1 & 1 & 9 \\
$15-18$ anos & 4 & 5 & 1 & 3 & 13 \\
$19-21$ anos & 1 & 1 & - & 1 & 3 \\
$>21$ anos & - & 1 & - & - & 1 \\
Total & 8 & 11 & 4 & 6 & 29 \\
\hline \hline
\end{tabular}

Dentre as 29 pessoas com hábito de beber, 18 participantes afirmaram que bebem somente aos finais de semana, 5 pessoas bebem aos finais de semana e alguns dias de semana e 6 pessoas não responderam.

Ao serem questionados sobre os fatores que influenciam positivamente para beber, a alternativa de "beber após um dia cansativo" foi assinalada 7 vezes, "durante a semana de prova" foi assinalada 3 vezes, "após uma semana de prova" foi marcada 13 vezes e "beber durante festas" foi apontada 16 vezes.

TABELA 3-Comportamento de risco ou influência social devido ao álcool
A tabela 3 compara os comportamentos de risco ou influência social relatados por estudantes devido ao uso de álcool, é importante destacar que um mesmo aluno pode responder mais de um item. Neste quesito, os 29 estudantes com hábito de beber responderam sim 64 vezes para os comportamentos de risco descritos na tabela 3 , enquanto os outros 47 estudantes não têm o hábito, já experimentaram álcool e tiveram comportamento de risco e influência social em 21 tipos de situações.

\begin{tabular}{lc|c}
\hline \hline & Sim - Com hábito & Sim - Sem hábito \\
\hline \hline Embriagar & 26 & 12 \\
Dirigir & 12 & 5 \\
Envolver em brigas & 5 & - \\
Sofreu acidentes & 2 & 3 \\
Faltou a faculdade & 15 & 1 \\
Faltou o trabalho & 4 & 21 \\
\hline \hline TOTAL & 64 & \\
\hline \hline
\end{tabular}


Analisando cada grupo por período de curso individualmente, é possível observar que no primeiro período 1 pessoa nunca teve contato com álcool e 9 pessoas já tiveram contato, apenas 2 pessoas mantiveram o hábito de beber regularmente. No segundo período, 10 pessoas relataram contato com o álcool, 7 pessoas não mantiveram o hábito de beber e 3 pessoas têm o hábito de beber, aos finais de semana.

No terceiro período, 10 pessoas já consumiram álcool, 7 não têm hábito de beber e 3 pessoas bebem rotineiramente. No quarto período, 2 pessoas nunca tiveram contato com álcool, 8 já tiveram contato com

\section{DISCUSSÃO}

Observou-se que mulheres e homens tiveram o primeiro contato com o álcool entre 15-18 anos, sendo essa a idade em que $78 \%$ das mulheres experimentaram pela primeira vez e $44,8 \%$ dos homens também. É no período da adolescência que a população, independente do sexo, fica mais vulnerável ao primeiro contato com substâncias psicoativas lícitas ou ilícitas. Pois as mudanças que ocorrem nessa época podem provocar incertezas, crise de identidade e instabilidade emocional. Muitas vezes, o uso de drogas, como o álcool, ocorre para solucionar questões que poderiam ser resolvidas de outras formas. 5

Foi identificada uma prematuridade nos hábitos masculinos já que $41,3 \%$ começaram a beber com menos de 14 anos (10,3\% com menos de 12 anos). Enquanto isso, 10,6\% das mulheres tiveram o contato com menos de 14 anos, 4\% com menos de 12. Esse resultado vai de encontro ao que foi mostrado por Malta que dividiu as crianças em faixas etárias e analisou qual sexo mais experimentou bebi- bebidas alcoólicas, em que 4 pessoas mantiveram o hábito de beber. No quinto período, 10 pessoas afirmaram contato com álcool, 5 não mantiveram o hábito e 5 mantiveram o hábito.

No sexto período, 1 pessoa afirmou nunca ter bebido, 9 afirmaram contato pelo menos em algum momento, destas, 5 não têm hábito e 4 bebem costumeiramente. No sétimo período, 10 pessoas afirmaram já ter feito uso de álcool alguma vez, 4 mantiveram o hábito e 6 não mantiveram. No oitavo período, 10 pessoas afirmaram ter tido contato com álcool, 9 possuem o hábito de beber, e 1 não tem.

das alcoólicas em cada faixa. Foi observado que entre 12-13 anos as meninas eram maioria, já a partir dos 14 anos os meninos predominavam 6 .

O consumo de álcool na adolescência é prejudicial para o desenvolvimento neuropsicomotor, está mais associado a morte do que todas as substâncias psicoativas em conjunto, aumenta a chance de violência sexual, tanto para o agressor quanto para a vítima, aumenta a taxa de atividade sexual sem proteção e o consumo está associado a prejuízos acadêmicos e distúrbios de formação de identidade. ${ }^{7}$

$\mathrm{O}$ ambiente onde as mulheres mais frequentemente tiveram o primeiro contato foi na casa de amigos (57,4\%), enquanto os homens, 37,9\%, experimentaram pela primeira vez mais em festas. É importante falar que grande porcentagem dos homens, 34\%, experimentou pela primeira vez junto dos pais ou com familiares, enquanto $21 \%$ das mulheres começaram nesse ambiente. Isso pode ser justificado pelas meninas esconderem mais 
os primeiros contatos com o álcool, enquanto os meninos não se submetem a isso. ${ }^{6}$ Provavelmente existe um fator cultural e patriarcal nesse fato, mas mais pesquisas são necessárias para a confirmação concreta.

Durante a análise das pessoas que já experimentaram álcool, 55,2\% não têm hábito de beber. Ao comparar, ao longo dos períodos, a prevalência das pessoas com hábito de beber, é possível observar que é mais comum esse hábito nos períodos mais avançados. A média de alunos que costumam beber nos 4 primeiros períodos é de 4,25 por período, considerando que foram estudados 10 de cada período acadêmico. Nos 4 períodos posteriores esse média subiu para 6,25 . Isso significa que nos 4 primeiros períodos o mais comum eram os alunos que não tinham hábito de consumir bebida alcoólica, enquanto nos 4 períodos seguintes os alunos que bebiam corriqueiramente eram maioria. Esse achado sugere que os estudantes estão adquirindo o hábito de beber ao longo da graduação de medicina, entretanto um maior grupo amostral iria solidificar esse achado.

Quanto aos estudantes que afirmaram não terem costume de beber, metade deles relataram beber uma ou duas doses, cerca de $30 \%$ deles bebem entre 3 a 5 doses e próximo de $20 \%$ deles bebem mais de 5 doses.

Das pessoas que não têm hábito de beber, mas já consumiram álcool, cerca de $71 \%$ já chegou ao estado de embriaguez. Esses acadêmicos pertencem a um padrão de consumo importante, que são as pessoas que bebem esporadicamente, mas consomem quantias consideráveis de álcool quando bebem, no processo conhecido como "binge drinking". A pessoa que realiza binge drinking está em um grupo de risco, pois elas tendem a consumir mais frequentemente ao longo do tempo e podem estar sujeitas a problemas de saúde nesses episódios ou em consequência deles. ${ }^{8}$

O comportamento de risco mais co- mum apresentado pelas pessoas sem costume de beber foi dirigir sob efeito de álcool (9,5\%). O segundo mais comum foi faltar a faculdade $7 \%$. Entretanto, uma delas afirmou ter relação abusiva com o álcool. Esses comportamentos serão melhor discutidos em seguida.

Mudando o foco para as pessoas que têm hábito de beber, elas compõem 44,8\% dos estudantes que já experimentaram álcool. A maioria bebe mais de 5 doses (74\%). Entretanto, ao analisar o padrão de consumo apenas dos primeiros 4 períodos, é possível notar que metade dos alunos bebem mais de 5 doses e a outra metade bebe entre 3 e 5 doses. Já 85,7\% dos alunos nos 4 períodos seguintes bebem mais de 5 doses, enquanto o restante bebe entre 3 e 5 doses. Esse dado sugere que os alunos nos períodos mais avançados ingerem maior quantidade de álcool. Ao somar isso ao achado de que mais pessoas têm costume de beber nos períodos mais avançados, a hipótese de que os alunos bebem mais ao longo da formação é sedimentada.

Mais de $82 \%$ dos estudantes que possuem hábito de beber já ficaram embriagadas. O comportamento de risco mais achado foi o absenteísmo acadêmico que chegou a $41,2 \%$, logo em seguida foi a direção sob efeito de álcool com 38,2\% dos estudantes. 5,8\% dos alunos que têm o costume de beber afirmaram possuir relação abusiva com álcool.

Logo o absenteísmo acadêmico foi a complicação, em decorrência do consumo de álcool mais comum, tanto entre pessoas com hábito de beber ou sem. Isso é algo subvalorizado, pois as faltas acadêmicas, a desatenção dentro da sala de aula e a desmotivação atrapalham o desenvolvimento acadêmico desse aluno. 9

Com relação à frequência de consumo, a maioria dos estudantes com hábito de beber marcou que consumiam a bebida nos finais de semana (73\%), o restante afirmou beber também em alguns dias da semana. 
Para estudar quais foram os fatores que propiciam o hábito de beber, foi perguntado quais as situações que levavam os alunos beber mais. 70\% dos estudantes que têm costume de beber afirmaram que as festas da turma favorecem o consumo. Isso ocorre porque os alunos são estimulados a beber nesses momentos. A bebida assume um caráter não só de integração, mas também de socialização desse estudante no universo acadêmico, tido como formador do jovem para a vida adulta. ${ }^{10}$

A semana de prova também favoreceu o consumo para $66 \%$ dos estudantes. 0 estresse provoca reações hormonais que desencadeiam no organismo claras modificações físicas e emocionais, produzindo uma série de sintomas emocionais tais como apatia, depressão, desânimo, sensação de desalento, hipersensibilidade emotiva, raiva, ira, irritabilidade e ansiedade. Sintomas que facilmente podem ser aliviados imediatamente pelo consumo de bebidas alcoólicas. Logo, o estresse tanto pode levar ao uso abusivo de álcool e ao alcoolismo, como o alcoolismo deixa o usuário mais vulnerável ao estresse. ${ }^{11}$ Outro dado que corrobora com isso é que 30\% afirmaram beber mais depois de dias cansativos.

Entre todos os alunos, apenas 3 deles afirmaram possuir relação abusiva com o álco- ol, 2 deles têm costume de beber e 1 não. Esse valor é aproximadamente $4 \%$ das pessoas que já experimentaram álcool. É importante saber que essa é uma avaliação pessoal, pois se forem utilizados os parâmetros de estudos que consideram uso abusivo como ingestão de 5 drinques por noite, encontra-se que $35 \%$ das pessoas estudadas fizeram uso abusivo. ${ }^{12}$

Portanto, muitos pacientes consideram as atitudes com o álcool como abusiva, mesmo utilizando grandes volumes de álcool, isso se explica pela bebida ser considera uma substância banal. ${ }^{10}$ Um exemplo desse comportamento foi o aluno que bebia mais de 5 doses, já se embriagou, dirigiu alcoolizado, faltou aula e se envolveu em brigas devido a bebida.

Quando questionados sobre a alteração do padrão de consumo de bebida alcoólica, $14,7 \%$ afirmaram que diminuíram o consumo ao longo dos períodos, enquanto $22 \%$ responderam que estão consumindo mais álcool no decorrer da graduação. Os $63,3 \%$ restantes não mudaram o padrão de consumo. Esse resultado significa que os estudantes acham que não estão bebendo mais. Entretanto, foi visto que, ao longo dos períodos, as pessoas tendem a ter mais o hábito de beber e consomem maior volume de bebidas.

\section{CONCLUSÃO}

A maioria dos estudantes da Famene teve/tem o primeiro contato com o álcool na adolescência e isso é algo possivelmente prejudicial para esses jovens. A maioria dos alunos não tem hábito de beber, mas no decorrer do curso, as pessoas que costumam beber se tornam predominantes, o que sugere que os estudantes de medicina estão bebendo mais ao longo da graduação. Entretanto, um estu- do que acompanhasse grupos de alunos, desde o primeiro período até o final da formação acadêmica, poderia solidificar os achados desse artigo. Os alunos que não possuem hábito de beber geralmente bebem poucas doses, porém alguns deles bebem grandes volumes e fazem uso abusivo do álcool. Já os estudantes que tem costume de beber consomem mais de 5 doses, que é considerado uso abusivo por 
alguns estudos. O maior favorecedor do consumo foram as festas da turma e o estresse decorrente das épocas de provas, isso é um dado importante que precisa ser trabalhado com medidas de educação em saúde e conscientização, para combater as complicações em decorrência do etilismo. O fator de risco mais comumente encontrado foi o absenteísmo acadêmico e depois a direção sob efeito de entorpecentes. Entretanto, apesar de tudo o que foi exposto, a maioria dos estudantes acham que seu consumo não modificou ao longo do curso, reforçando a necessidade de medidas de conscientização.

\title{
ANALYSIS OF THE USE OF ALCOHOLIC BEVERAGES BY STUDENTS OF MEDICINE OF A PRIVATE INSTITUTION
}

\begin{abstract}
Excessive use of alcohol can cause harm to the most diverse spheres of human life, being a risk factor for various health problems, as well as making it easier for users to adopt risky behaviors. There are some population groups that are more susceptible to alcohol abuse, such as high-stress professions and people under high stress. The purpose of this article is to analyze the pattern of alcohol use among medical students. This is a descriptive field research with a quantitative approach. The population is all 815 students between the first and eighth years of the Nova Esperança Medical School. The sample was calculated with a sampling error of $11 \%$ and the confidence level of $95 \%$, reaching the result of 77 students, this value was rounded to 80 with the purpose of using 10 students from each period and obtaining a more representative sample of the populational group. A semi-structured questionnaire was used to collect data. According to the results, $93.75 \%$ of the participants reported having tasted alcoholic beverages, $44 \%$ of which had the drinking habit. $78 \%$ of the women and $44.8 \%$ of the men had the first contact between $15-18$ years. The average number of students without a drinking habit is 6.25 in the first 4 periods and 4.25 in the last 4 , suggesting an increase in the indices of students with drinking habits over the periods. $71 \%$ of the people without habit have already reached the state of drunkenness, predisposing risk behavior. Among those with a habit, $82 \%$ were already intoxicated. Of these, $41.2 \%$ practiced academic absenteeism, 38.2\% drove under alcohol and only 5.8\% said they had an abusive relationship with alcohol, $70 \%$ stated that class parties favor consumption, and test week, for $66 \%$ of students. It is concluded that generally the first contact with alcohol is in adolescence and, although the vast majority have already tried alcoholic beverages, most of the students did not keep the drinking habit. It was possible to observe that the drinking habit is more prevalent in students of more advanced periods than the students that more recently entered the course.
\end{abstract}

KEYWORDS: Alcoholism; Medical students; Epidemiology.

\section{REFERÊNCIAS}

1. Veiga LDB, Santos VC, Santos MG, Ribeiro JF, Amaral ASN, Nery AA, et al. Prevalência e fatores associados à experimentação e ao consumo de bebidas alcoólicas entre adolescentes escolares. 


\section{revista de
ciências da saúdes ESPERANÇA}

Cad. saúde colet. 2016 Sep; 24(3):368-375.

2. Araújo $P$, Carvalho MGN, van Weelden M, Lourenço $B$, Queiroz LB, Silva CA. Substance misuse and sexual function in adolescents with chronic diseases. Rev. paul. pediatr. 2016 Sep; 34( 3 ): 323-329.

3. Oliveira SKM, Sousa ÁAD, Cavalcanti SL, Taveira MGMM, Correia DS, Freitas D. Uso de Bebidas Alcoólicas entre Acadêmicos da Área de Saúde. Revista Brasileira de Educação Médica. 2016 Sep; 40(3), 446-451.

4. Dázio EMR, Zago MMF, Fava SMCL. Use of alcohol and other drugs among male university students and its meanings. Rev. esc. enferm. USP [Internet]. 2016 Oct; 50(5): 785-791.

5. Vargas Divane de, Soares Janaina, Leon Erika, Pereira Caroline Figueira, Ponce Talita Dutra. O primeiro contato com as drogas: análise do prontuário de mulheres atendidas em um serviço especializado. Saúde debate [Internet]. 2015 Sep [cited 2017 June 10]; 39(106 ): 782-791.

6. Malta Deborah Carvalho, et al. Prevalência do consumo de álcool e drogas entre adolescentes: análise dos dados da Pesquisa Nacional de Saúde Escolar. Rev. bras. epidemiol. [Internet]. 2011 Sep; 14(1): 136-146. Disponível em: http:// www.scielo.br/scielo.php?script=sci_arttext\&pi-

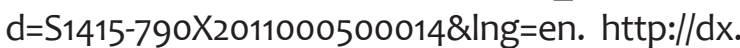
doi.org/10.1590/S1415-790X2011000500014.
7. Pechanskya, Flavio, Claudia Maciel Szobota, and Sandra Scivolettob. "Uso de álcool entre adolescentes: conceitos, características epidemiológicas e fatores etiopatogênicos Alcohol use among adolescents: concepts, epidemiological characteristics and etiopatogenic." Rev Bras Psiquiatr 26. Supl I (2004): 14-17.

8. Jang, Joy Bohyun, et al. "Frequent binge drinking among US adolescents, 1991 to 2015." Pediatrics (2017): e20164023.

9. Miranda, Gilberto José, Tamires Sousa Araujo, and Izabelle Almeida Marcelino. "O absenteísmo acadêmico e suas consequências mais óbvias." Revista Gestão Universitária na América Latina-GUAL 10.1 (2017).

10. no Trabalho, A. Inserção do Psicólogo. "de Prevenção ao Abuso de Álcool e Outras Drogas." Psicologia ciência e profissão 24.1 (2004): 108-115.

11 Jora, Natalia, Thais Roberto Magalhães, Josélia Benedita Carneiro Domingos, \& Sandra Cristina Pillon. "Campanha saúde na estrada: avaliação do padrão de consumo de álcool e do estresse." Revista Eletrônica de Enfermagem [Online], 12.1 (2010): sem paginação Web. 12 Jun. 2017

12. Barros Mauro V G de, Nahas Markus V. Comportamentos de risco, auto-avaliação do nível de saúde e percepção de estresse entre trabalhadores da indústria. Rev. Saúde Pública [Internet]. 2001 Dec [cited 2017 June 12] ; 35 ( 6 ): 554-563. 